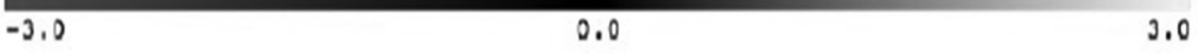

Z-score

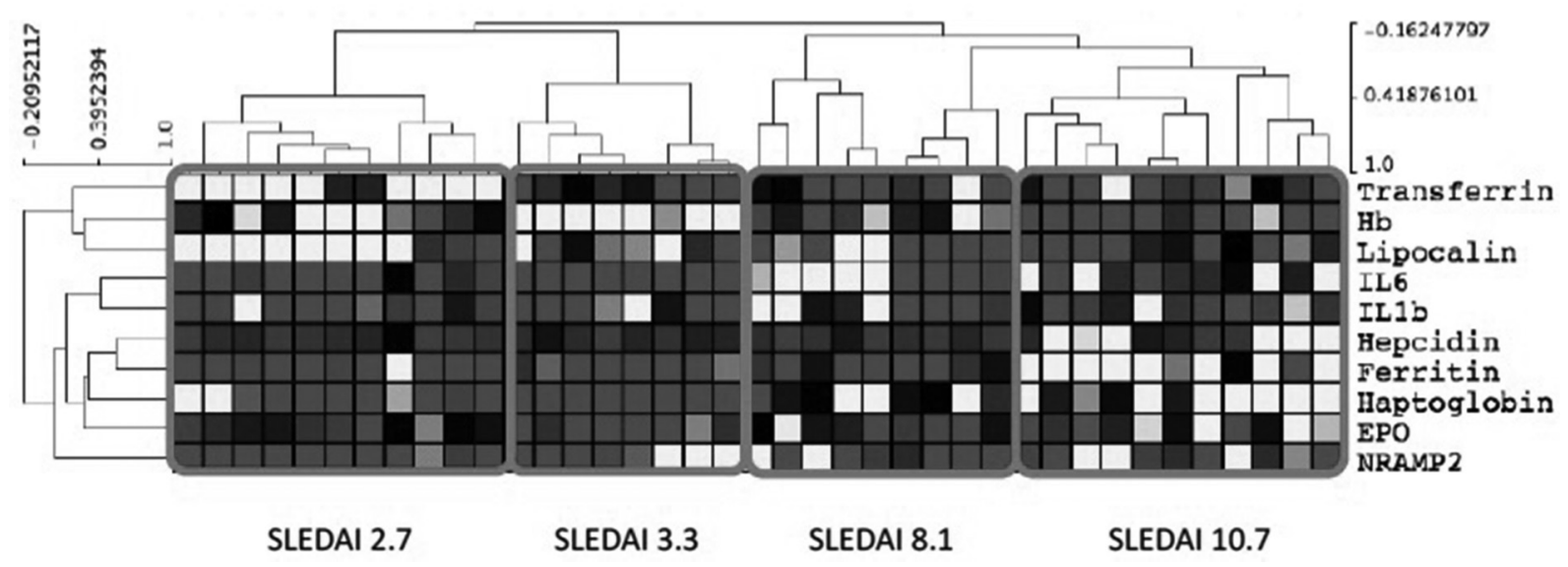

Abstract P42 Figure 1 Heatmap representation of cluster analysis of key iron mediators in SLE. Normalised data is presented as Z-score with -3.0

spite of immune activation. Hierarchical clustering is presented in figure 1. Cluster analysis demonstrates that the group with the highest mean SLEDAI-2K (10.7) had lower $\mathrm{Hb}$, transferrin and LCN2 in addition to elevated IL1 $\beta$, IL6 and hepcidin compared with those with lower SLEDAI-2K. Elevated haptoglobin levels were seen in those in the higher disease activity groups, suggesting that haemolysis was an unlikely cause for anaemia.

Conclusions The findings of this study suggest increased lupus disease activity results in abnormal iron homeostasis through impaired cellular iron import (via reduced transferrin), a lack of stored iron release (under the actions of elevated hepcidin) and reduced iron sequestration by LCN2, which may represent a novel cause of non-haemolytic anaemia.

Acknowledgements This work was supported by Versus Arthritis (549143) and LUPUS UK (176255).

\section{P43 SERUM S100A8/A9 CONCENTRATIONS ARE ASSOCIATED WITH NEUROPSYCHIATRIC INVOLVEMENT AND FATIGUE IN SLE}

${ }^{1}$ Kristoffer Alexander Zervides, ${ }^{1}$ Andreas Jern, ${ }^{2}$ Jessica Nystedt, ${ }^{2}$ Petra Nilsson, ${ }^{3}$ Pia C Sundgren, ${ }^{4}$ Birgitta Gullstrand, ${ }^{1}$ Anders A Bengtsson, ${ }^{1}$ Andreas Jönsen. ${ }^{1}$ Dept. of Clinical Sciences, Rheumatology, Lund University, Skåne University Hospital, Lund; '²Dept. of Clinical Sciences, Neurology, Lund University, Skåne University Hospital, Lund; ${ }^{3}$ Dept. of Clinical Sciences, Diagnostic Radiology, Lund University, Skåne University Hospital, Lund; ${ }^{4}$ Dept. of Laboratory Medicine, Clinical Immunology, Lund University, Skåne University Hospital, Lund, Sweden

\subsection{6/lupus-2020-eurolupus. 91}

Background/Purpose Neuropsychiatric (NP) involvement and fatigue are both major problems in SLE. S100A8/A9 is a marker of inflammation, which responds to therapy in SLE patients. S100A8/A9 is expressed in the CNS. We investigated S100A8/A9 in relation to NPSLE and fatigue.

Methods In this cross-sectional study we used ELISA (Bhulmann MRP8/14 ELISA kit, Switzerland) to measure the concentration of S100A8/A9 in serum in 72 SLE patients and 26 healthy controls and in cerebrospinal fluid (CSF) in 33 SLE patients. NP involvement was determined according to ACR case definitions for NPSLE. An MRI was performed in SLE patients and controls assessing white matter abnormalities and cerebral atrophy. Measurements of fatigue were performed using the Fatigue Severity Scale (FSS) and the Visual Analogue Scale $(100 \mathrm{~mm})$ (VAS). Statistical calculations were performed using non-parametric methods.

Results In all, 72 female SLE patients (median age 38, range 18-52) and 26 female healthy controls (median age 40, range 23-52) were included in this study. Forty-four (61\%) patients had NP involvement. NPSLE patients had higher serum S100A8/A9 concentrations (median $1.40 \mu \mathrm{g} / \mathrm{ml}$ ) than the nonNPSLE patients (median $0.92 \mu \mathrm{g} / \mathrm{ml} ; \mathrm{p}=0.011$ ) and the control group (median $0.79 \mu \mathrm{g} / \mathrm{ml} ; \mathrm{p}=0.004$ ). Serum S100A8/A9 correlated with increased VAS fatigue in SLE patients $(\mathrm{r}=0.311 ; \mathrm{p}=0.008)$, but not with FSS $(\mathrm{r}=0.184 ; \mathrm{p}=0.124)$. Serum S100A8/A9 did not correlate with the extent of white matter lesions, atrophy of brain segments, or disease activity (SLEDAI-2K). S100A8/A9 was not detected in the CSF.

Conclusions Higher serum S100A8/A9 concentrations in NPSLE patients and patients with fatigue may indicate that S100A8/A9 is involved in the pathogenesis of these manifestations, although further investigation is needed.

\section{P44 URTICARIAL VASCULITIS IN CHILDHOOD ONSET SYSTEMIC LUPUS ERYTHEMATOSUS}

${ }^{1}$ Reem Abdwani, ${ }^{2}$ Safiya Al Abrawi, ${ }^{3}$ Ahmed Al Kamzari, ${ }^{4}$ Ibraheem Al Zakwani, ${ }^{5}$ Buthaina Al Masilhi. ${ }^{1}$ Child Health Dept., Sultan Qaboos University, Muscat; ${ }^{2}$ Child Health Dept., Royal Hospital, Muscat; ${ }^{3}$ Child Health Dept., Oman Medical Specialty Board, Muscat; ${ }^{4}$ Pharmacology Dept., Sultan Qaboos University Hospital, Muscat; ${ }^{5}$ Dermatology Dept., Sultan Qaboos University Hospital, Muscat, Oman

\subsection{6/lupus-2020-eurolupus.92}

Purpose Urticarial vasculitis (UV) is a rare type of vasculitis in children frequently, idiopathic, however, can be associated childhood onset SLE. The aim of the study is to describe the frequency of UV in CSLE and to compare the epidemiological, clinical and laboratory characteristics of cSLE stratified by the presence of UV in a cohort of patients of Arab ethnicity from Oman. 